\title{
Near-Earth magnetic signature of magnetospheric substorms and an improved substorm current model
}

\author{
P. Ritter and H. Lühr \\ Helmholtz-Zentrum Potsdam Deutsches GeoForschungsZentrum - GFZ, Telegrafenberg, 14473 Potsdam, Germany
}

Received: 4 March 2008 - Revised: 8 August 2008 - Accepted: 19 August 2008 - Published: 18 September 2008

\begin{abstract}
Based on a comprehensive catalogue with more than 4000 magnetospheric substorm entries from the years 2000-2005, the spatial distribution of the substorm-related magnetic signatures at mid and low latitudes around local midnight was investigated. Superposed epoch analysis of a larger number of recent observatory data from mid and low latitudes revealed a field strength increase that is consistent with the results of earlier studies. For the first time, the magnetic signature of the substorm current wedge formation is studied also in near-Earth satellite data from CHAMP. The average maximal deflection measured on board the satellite is smaller by a factor of 2 than that determined from ground observations. The recurrence frequency of substorms as well as the amplitude of their magnetic signature depends strongly on the prevailing magnetic activity. The observed average substorm-related magnetic field signatures cannot be described adequately by a simple current wedge model. A satisfactory agreement between model results and observations at satellite height and on ground can be achieved only if the current reconfiguration scenario combines four elements: (1) the gradual decrease of the tail lobe field, (2) the rerouting of a part of the cross-tail current through the ionosphere, (3) eastward ionospheric currents at low and mid latitudes driven by Region-2 field-aligned currents, and (4) a partial ring current connected to these Region-2 FACs.
\end{abstract}

Keywords. Ionosphere (Electric fields and currents; Ionosphere-magnetosphere interactions) - Magnetospheric physics (Storms and substorms)

\section{Introduction}

Substorms are regarded as an important part of the magnetospheric activity cycle. The loading by the solar wind is fol-

Correspondence to: P. Ritter

(pritter@gfz-potsdam.de) lowed by an impulsive unloading of energy previously stored in the magnetospheric tail. Different assumptions exist about the physical conditions that initiate a substorm. For example, the cross-tail current instability model postulates that plasma instabilities in the near-Earth region $\left(6-10 R_{E}\right)$ start the process causing a cross-tail current disruption and a divergence into the ionosphere via field-aligned currents (Lui, 1996). Alternatively, the near-Earth neutral line model predicts that the substorm is initiated at a further distance downtail $\left(>20 R_{E}\right)$ by reconnection (Baker et al., 1996). The disruption of crosstail currents is an intrinsic part of reconnection. In this model the region of disrupted cross-tail currents expands earthward. Common to both models is the formation of a substorm current wedge. In a large-scale reconfiguration the disrupted cross-tail current is rerouted along the field lines towards the Earth and closed in the high-latitude ionosphere on the night side. A schematic presentation of this current system was published by Clauer and McPherron (1974).

As a consequence the substorm magnetic energy previously stored in the tail is converted to kinetic energy of the plasma. The related tail lobe magnetic field reduction can be observed as a clear signature in magnetic field measurements at the Earth's surface. In an earlier statistical study Caan et al. (1975) attributed the field strength increase at mid latitudes of about $10 \mathrm{nT}$ subsequent to a substorm onset to the lobe magnetic field change. This observation was obtained using superposed epoch analysis. The substorm onset times of the twenty events considered in that study were defined by the first positive magnetic deflection evident in the night time sector around midnight. The onsets had been verified by observed tail lobe field fluctuations of the OGO 5 spacecraft. These early studies can be considered as a qualitative proof-of-concept of the envisaged substorm current configuration. They are not sufficient for assessing the details of the current systems. The question arises, whether the nearEarth magnetic signatures at other latitudes are also consistent with the substorm current model? Furthermore, can the

Published by Copernicus Publications on behalf of the European Geosciences Union. 

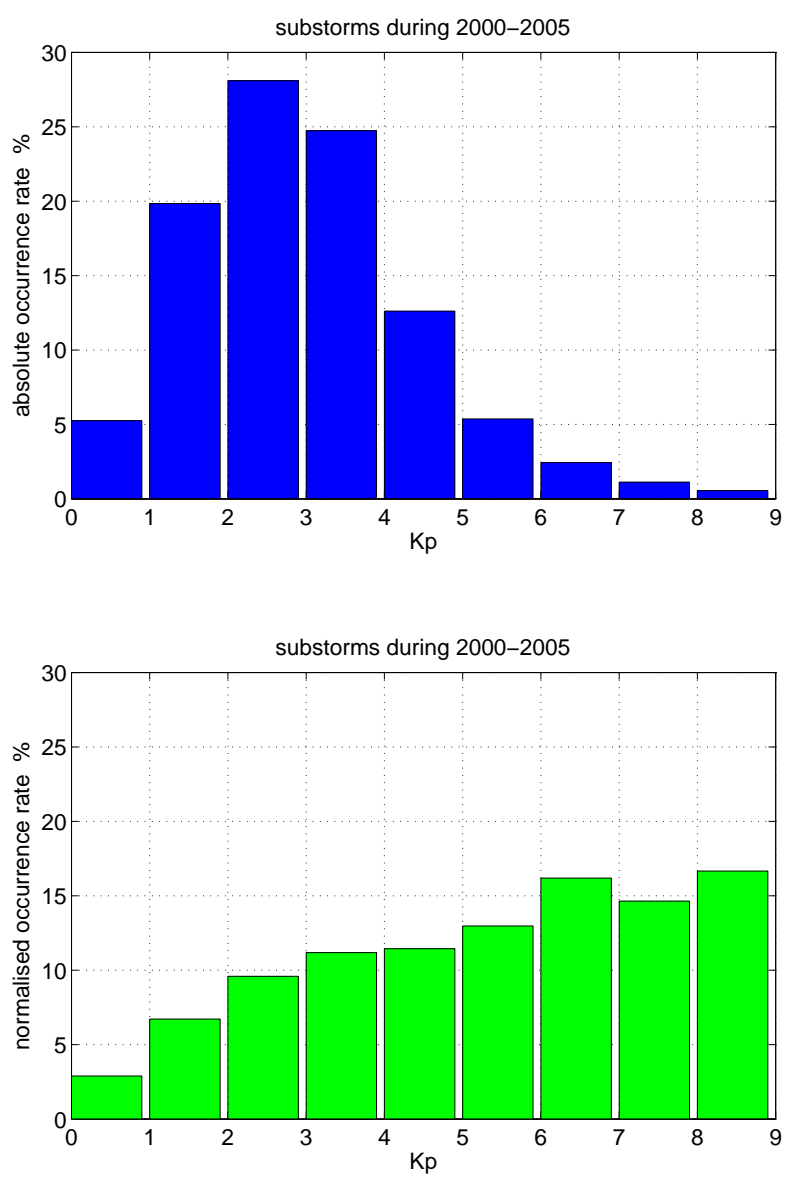

Fig. 1. Occurrence of substorms with respect to $K_{p}$. Top frame: percentage of substorms that occur at $K_{p}$ levels 0-9; bottom frame: substorm occurrence rate, normalised by the distribution of $K_{p}$ numbers during the entire time period covered by the onset cata$\log$ (May 2000-December 2005).

total current in the substorm loop be estimated from the lowlatitude ground signature?

The motivation for the study presented in this paper is to reproduce and consolidate the results of Caan et al. (1975) by using recordings from modern magnetic observatories and by employing the high-resolution magnetic field measurements of the CHAMP satellite. Another goal is to find out how the substorm-related signatures depend on magnetic activity. For the construction of high-degree geomagnetic field models typically CHAMP data from the night side are used. So far, the occurrence of substorms has not been taken into account for data selection. Thus, we intend to determine the average size of substorm signatures in CHAMP data at low and mid latitudes.

To achieve these goals we make use of the extensive substorm onset catalogue of Frey and Mende (2006). Statistically significant results for several aspects can be deduced from its more than 4000 entries of identified events. For the interpretation of the CHAMP magnetic field data we em- ployed the latest field models (e.g. Potsdam Magnetic Model of the Earth, POMME_3.1) to subtract all magnetic effects that are not related to a substorm.

In the following Sect. 2 we introduce the substorm cata$\log$ and observatories whose data were used in this study. In Sect. 3 we present the observations of a single substorm event starting with its detection by Far Ultraviolet (FUV) Images. We then show its magnetic field at high and low latitudes, and on board the satellite CHAMP. Section 4 describes the statistical studies that were performed with ground observations and with satellite data. In Sect. 5 we discuss the observational results and compare them with a simple and a more detailed substorm current model. The final conclusions in Sect. 6 summarize the observations and the design of the inferred current model.

\section{Data sets}

\subsection{IMAGE FUV - substorm catalog}

For this study we used a comprehensive catalog of substorm onset times that was compiled by Frey and Mende (2006) and spans the years 2000-2005. The substorm onset times (approximately 4200 events) were identified from the images of the far ultra-violet (FUV) instrument on board the IMAGE spacecraft, using the Wideband Imaging Camera (WIC) and the Spectrographic Imager Channel (SI-13). The conditions for substorm recognitions were threefold: i) a clear local brightening of the aurora; ii) its expansion to the poleward boundary of the auroral oval and a minimum azimuthal spread of 20 min in local time; iii) a gap of 30 min between events. The IMAGE spacecraft observed the auroras of the Northern Hemisphere in 2000-2003 and then moved to the Southern Hemisphere. In the Northern Hemisphere, 2760 events were identified, while in the Southern Hemisphere 1430 substorm onsets were detected.

When averaged over all seasons and years the mean substorm onset location is the same in both hemispheres: the average magnetic local time (MLT) for a substorm onset is $22.9 \mathrm{~h}$ and the average magnetic latitude is $66.4^{\circ}$, regardless of the different general magnetic activities of the two subsets (Wang et al., 2005, 2007). A look at the histograms in Fig. 1, top frame, shows that most substorms occur at $K_{p}<4$, whereas only few substorms can be expected for stronger magnetic activity. Periods of strong activity, however, occur less frequently - hence the relative occurrence rate of substorms shows a gentle increase with increasing $K_{p}$ number (bottom frame), with a plateau-like maximum of about $15 \%$ for $K_{p}>5$. This means on a global average there is an onset every $6 \mathrm{~h}$ during high activity periods. Locally, however, the occurrence frequency depends on the longitude (Wang and Lühr, 2007). 

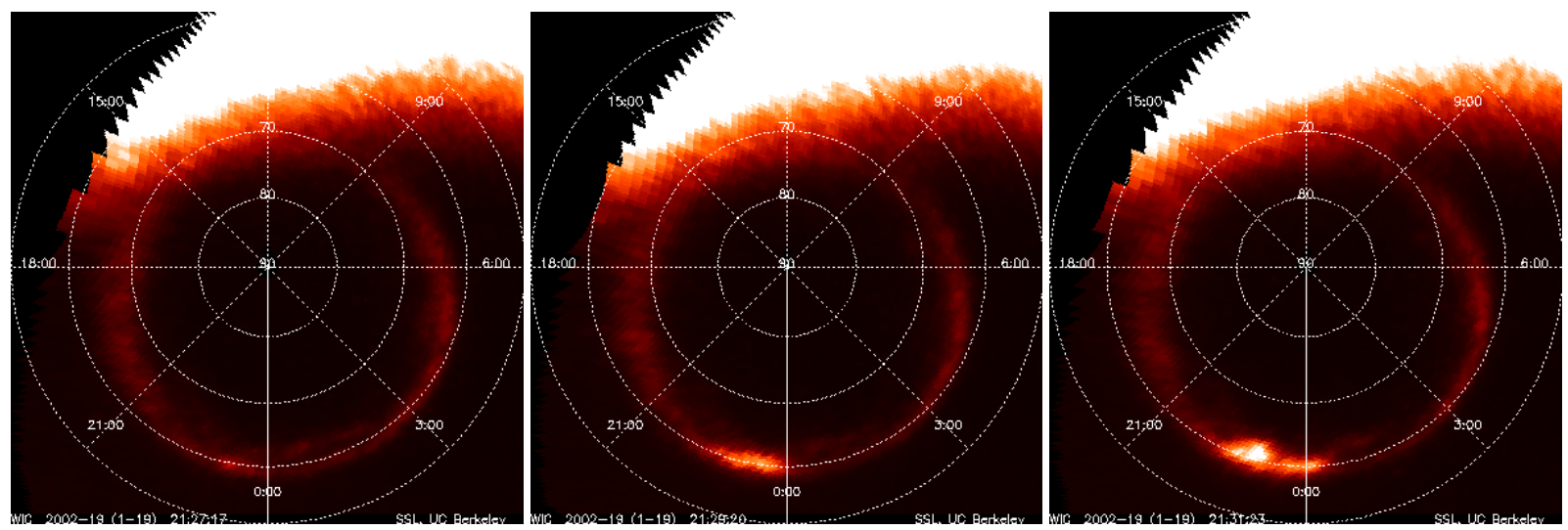

Fig. 2. Substorm onset detection by FUV images of the IMAGE spacecraft in the Mlat/MLT frame. Example substorm event on 19 January 2002 at 21:29:20 UT (middle frame). Onset location: 60.14 Mlat, 23:50 MLT. Times on the left and right frames are 21:27:17 and 21:31:23 UT, respectively.

\subsection{Magnetic observatories}

For the statistics of ground-based observations we used four observatories at similar magnetic longitudes (in the range $80^{\circ}-100^{\circ}$ Mlon). At low-latitudes, we employed the magnetic field data of the stations at Bangui (BNG), situated at $4.36^{\circ}$ Mlat, $90.8^{\circ}$ Mlon (dipole coordinates) and Tamanrasset (TAM), at $24.8^{\circ}$ Mlat , $81.6^{\circ}$ Mlon. Furthermore, at midlatitudes, data from L'Aquila (AQU), located at $42.45^{\circ}$ Mlat, 94. $6^{\circ}$ Mlon, and, further north, Niemegk (NGK), at 51.9 Mlat, $97.9^{\circ}$ Mlon, were considered.

\subsection{CHAMP orbits and data}

The satellite CHAMP (Reigber et al., 2002) was launched on 15 July 2000 into a circular, near-polar $\left(87.3^{\circ}\right.$ inclination) orbit. From its initial altitude at $456 \mathrm{~km}$ the orbit has decayed to about $350 \mathrm{~km}$ after 5 years. The orbital plane precesses at a rate of $1 \mathrm{~h}$ in local time (LT) per 11 days, thus visiting all local times within 131 days. The data used in this study are the scalar and vector magnetic field measurements of the Overhauser (OVM) and the Fluxgate Magnetometer, (FGM), respectively, with a resolution of $0.1 \mathrm{nT}$. The vector data are calibrated routinely with respect to the absolute scalar readings.

\subsection{Magnetic coordinates}

We use the following coordinate systems to describe and compare data and measurement locations: the locations of the four mid- and low-latitude observatories, which respond mainly to magnetospheric currents, are given in dipole coordinates. The coordinates of the five IMAGE magnetometer stations at high latitudes, responding to ionospheric currents, as well as the substorm onset locations identified by IMAGE FUV are given in corrected geomagnetic (cgm) coordinates (Richmond, 1995). The horizontal magnetic field, $H$, of the ground observations is compared to the three components of the CHAMP observations in dipole coordinates. When stacking the CHAMP data in the superposed epoch analysis we used the cgm system for the positions of the satellite readings.

\section{Example of a substorm event}

In the following we present as an example the observations of a single substorm event at the IMAGE spacecraft, at high and low latitude ground stations and at the CHAMP satellite.

\subsection{IMAGE FUV auroral observations}

Figure 2 shows the auroral images of the detection of a substorm event. Here, three consecutive snapshots, spaced by $2 \mathrm{~min}$, show the brightening and expansion of the aurora. The onset time was identified as 21:29:20 UT on 19 January 2002 in the middle plot. The onset location in the Mlat/MLT frame is at $60.14^{\circ}$ Mlat, 23:50 MLT.

\subsection{IMAGE magnetometer network - high latitudes}

The same event as identified with the IMAGE-FUV camera in Fig. 2 is clearly visible in the ground-based magnetometer readings of high latitude stations in the Northern Hemisphere. Figure 3 shows time-series of the X-component of five stations of the IMAGE Magnetometer Network in Scandinavia (Lühr et al., 1996). At this network the local midnight is 21:30 UT, hence convenient for the observation of the example substorm. Baselines were determined and subtracted using the advanced approach of Sillanpää et al. (2004). Negative deflections associated with the substorm electrojet are observed first at OUJ, the station closest to the onset latitude. They start already a few minutes before the reported onset time, 21:29 UT. Towards higher latitudes 


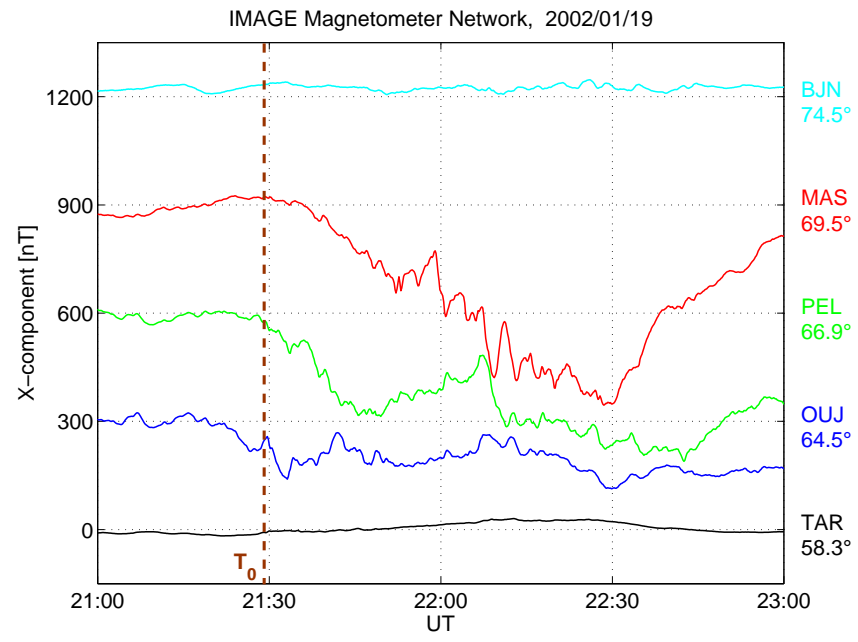

Fig. 3. Substorm signature at IMAGE magnetometer network stations BJN $\left(74.50^{\circ}\right.$ Mlat), MAS $\left(69.46^{\circ}\right)$, PEL $\left(66.90^{\circ}\right)$, OUJ $\left(64.52^{\circ}\right.$, TAR $\left.\left(58.26^{\circ}\right)\right)$. Same event as in Fig. 2. The reported onset time is marked by the vertical dashed line. Station OUJ is closest to the onset latitude.

the initiation starts later, but the electrojet strength increases. This is consistent with the poleward expansion of the substorm current closure. The largest excursions of more than $-500 \mathrm{nT}$ are observed at MAS, about $9^{\circ}$ north of the onset latitude, at 22:30 UT. Station BJN, located at $74.5^{\circ}$, does not display any magnetic signature due to a current increase, indicating that it is located too far north of the substorm electrojet.

\subsection{Observatory BNG - low latitudes}

At mid and low latitudes the magnetic signature of a substorm is expected to be generated by the distant disruption of the cross-tail current during the current wedge formation (Caan et al., 1975). This "missing" tail current starts at a distance of about 6-12 $R_{E}$, and accordingly, the effect of the tail lobe magnetic field variation at the Earth's surface is only of the order of some $10 \mathrm{nT}$ (e.g. Lopez et al., 1990). Figure 4 shows the magnetic $H$ component for the same event as introduced in Fig. 2, measured at the near-equator observatory Bangui. From the recorded data a recent main field model was subtracted using POMME-3 (Maus et al., 2006) and, additionally, the residuals were corrected for large scale magnetospheric variations with the help of the $D_{s t}$ index. The local time at BNG is $22.7 \mathrm{~h}$, i.e. close to the average substorm onset time on the night side. The reported onset time, $T_{0}$, is marked by an asterisk on the curve. After an amplitude drop of approximately $8 \mathrm{nT}$ during the half hour before the onset time the amplitude of the horizontal component increases by about $20 \mathrm{nT}$ within $1 \mathrm{~h}$ after $T_{0}$. The magnetic field enhancement lasts for a time span of $1 \mathrm{~h} 15 \mathrm{~min}$ and peaks at the same time (22:30 UT) as the electrojet strength in the auroral zone (cf. MAS, Fig. 3).

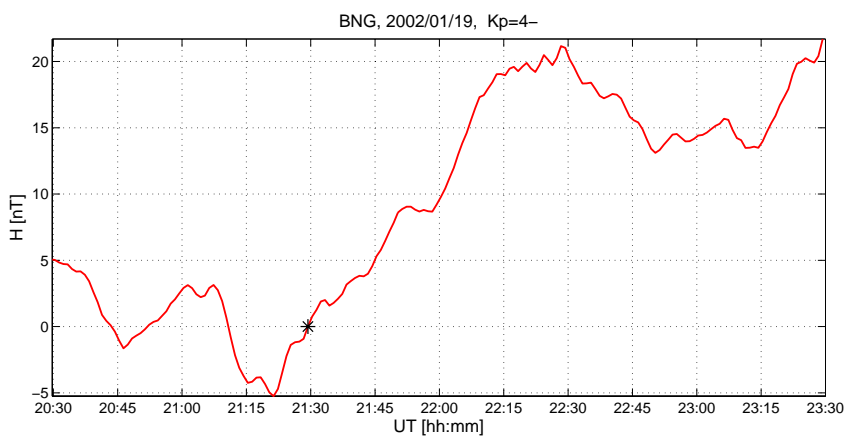

Fig. 4. Substorm signature at the observatory BNG $\left(4.36^{\circ}\right.$ Mlat $)$, same event as in Fig. 2. The onset time is marked by an asterisk.

\subsection{Satellite - CHAMP}

The same event as presented above can be observed in the CHAMP satellite data. The top frame of Fig. 5 shows the residuals of the magnetic field magnitude, $d F$, of three consecutive orbits. The satellite readings are corrected for main field (POMME-3, Maus et al., 2006), for crustal field (MF-5 model, Maus at al., 2007) and for ring current effects parameterized by the $D_{s t}$ index. Coming from the dayside equator on the left side of the frame the CHAMP satellite passes the North Pole (NP), crosses the magnetic equator in the middle of the frame and passes the South Pole (SP) on the right side. The plot below gives the respective local time at the satellite position. Again, the asterisk on the red curve marks the onset time of the substorm event. Here, it marks the location and local time of the satellite when the brightening of the aurora occurred. This central orbit is plotted in red, whereas the previous orbit is blue and the subsequent orbit is coloured green. Approximately $10 \mathrm{~min}$ after the substorm onset time the satellite passes the North Pole and descends on the night side towards the South Pole. Within the latitude interval $\left( \pm 50^{\circ}\right)$ the residuals of the red curve on the nightside are shifted upward by about $10 \mathrm{nT}$ compared to the blue curve at these latitudes, that was attained from measurements before the event. The positive shift is still evident during the subsequent orbit (green curve), ca. $1.5 \mathrm{~h}$ later (23:30 UT).

Figure 6 shows the difference between $d F$ measured before (blue curve) and after the substorm onset (red curve) in the latitude range $-50^{\circ}$ to $50^{\circ}$ on the night side. At higher latitudes CHAMP quickly leaves the local time sector of interest. The resulting amplitude shift seems to be caused by the current redistribution in the tail lobe, as it is observed by a near-Earth orbiting satellite. The maximum deflection, $13 \mathrm{nT}$, is less strong than the signature measured at the surface. Note that the sampling of the curve in Fig. 6 takes place from right to left. ie. northern to Southern Hemisphere. The large amplitude changes are caused by a mixture of temporal and spatial variations in account of the orbit separation of $93 \mathrm{~min}$. 


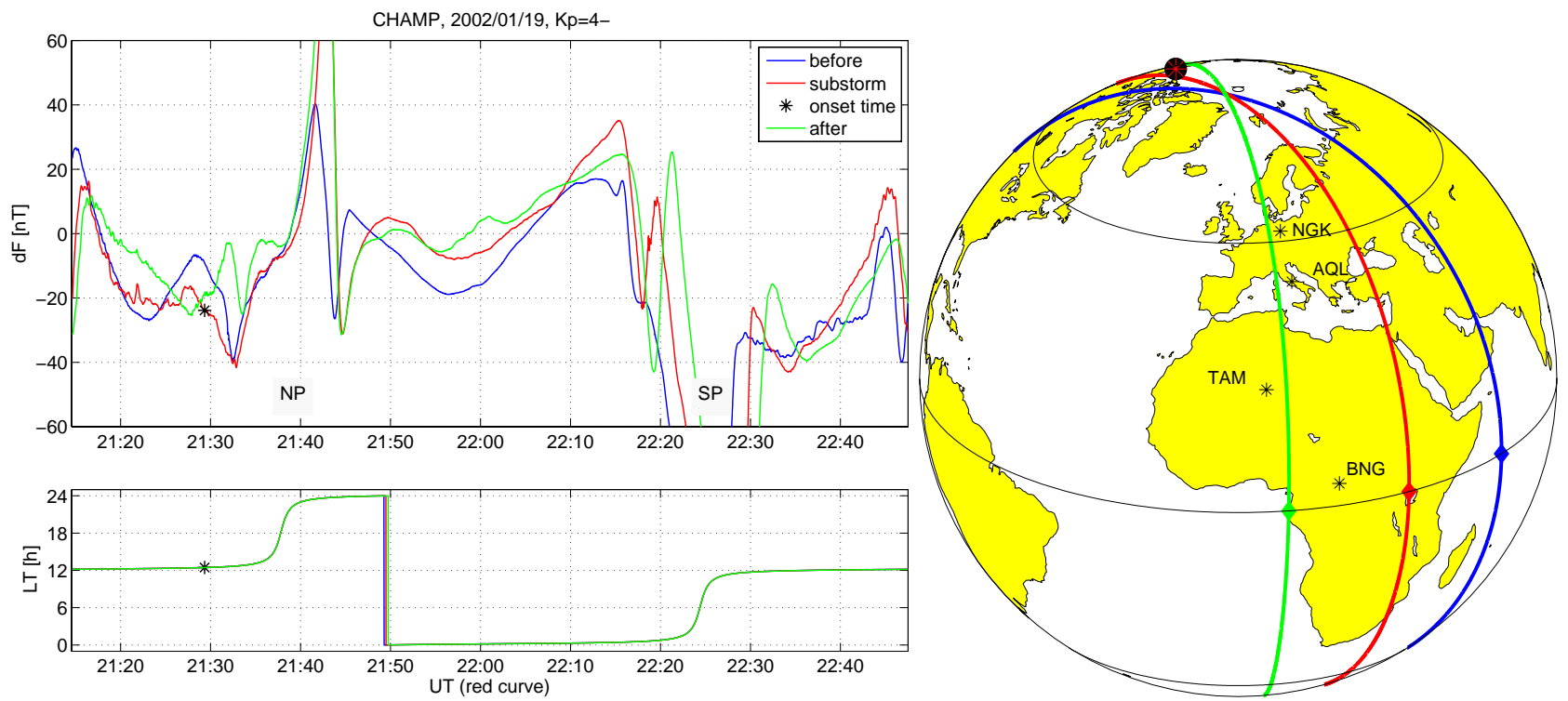

Fig. 5. Scalar magnetic field residuals, dF, of 3 consecutive CHAMP orbits, grouped around the event picked in Fig. 2. Each curve shows the magnetic recordings along a complete orbit starting northbound at the dayside equator. The UT time is valid for the middle (red) curve. The previous orbit is marked blue, the subsequent one green. The substorm onset takes place during the "red" orbit - it is marked by an asterisk.

\section{Statistical analysis}

In order to assess the significance of the single event results and to reproduce the observations at mid latitudes by Caan et al. (1975) with data from today's modern instrumentation and with the large number of substorm events available in the catalog of Frey and Mende (2006) we investigate the magnetic signature at the four observatories listed in Sect. 2.1 and at the near-Earth orbiting satellite CHAMP.

\subsection{Ground observations - BNG, TAM, AQU and NGK}

We start by looking into magnetic observations at Bangui Observatory (BNG). As this station is located not far from the magnetic equator and away from the complex polar current systems, one may expect to see the substorm magnetic signature most clearly during nighttime. To obtain a picture of the average dynamics of the magnetic field variations around a substorm onset, $T_{0}$, a superposed epoch analysis was performed. For that purpose all observations of a time span of $1 \mathrm{~h}$ before until up to $4 \mathrm{~h}$ after $T_{0}$ that occurred at night between 22:00 and 02:00 LT were stacked (607 events). Figure 7 shows the mean deflection of the $H$-component at BNG of all those 607 substorm events as a solid blue curve, regardless of magnetic activity. All time series were corrected for baselines as described for the example event in Fig. 4 of Sect. 3.3, and leveled to zero at the onset time, $T_{0}$.

As can be seen in the histogram in Fig. 1, substorms occur at all levels of magnetic activity, mostly at low and moderate $K_{p}$ values $<4$. The level of activity, however, is likely to influence the amplitude of the recorded magnetic field change.

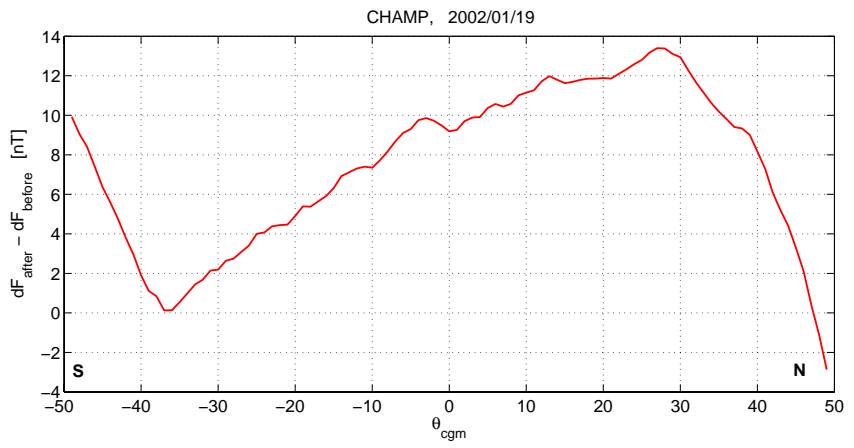

Fig. 6. Difference of the CHAMP orbits before and after the substorm onset on 19 January 2002.

Therefore the dataset used for the superposed epoch analysis was divided into two parts according to magnetic activity. The green curves in Fig. 7 reflect the signature for very low activity, when $K_{p}$ is smaller or equal 2 (164 events), whereas the red curves present the signature for $K_{p}$-values larger than 2 (443 events). The dashed lines mark the $68.2 \%$ confidence intervals of the respective mean curves. All curves show a distinct amplitude drop before the onset time, $T_{0}$, reflecting an intensification of the cross-tail current before the breakup. The minimum amplitude is strongest for the red, "active" curve. After the onset time the current wedge signature is marked by a pronounced enhancement of the horizontal magnetic field for about $1.5-2 \mathrm{~h}$. For all levels of activity the maximum of $H$ is reached at approximately $40 \mathrm{~min}$ after $T_{0}$. As expected, the maximum amplitude depends on 


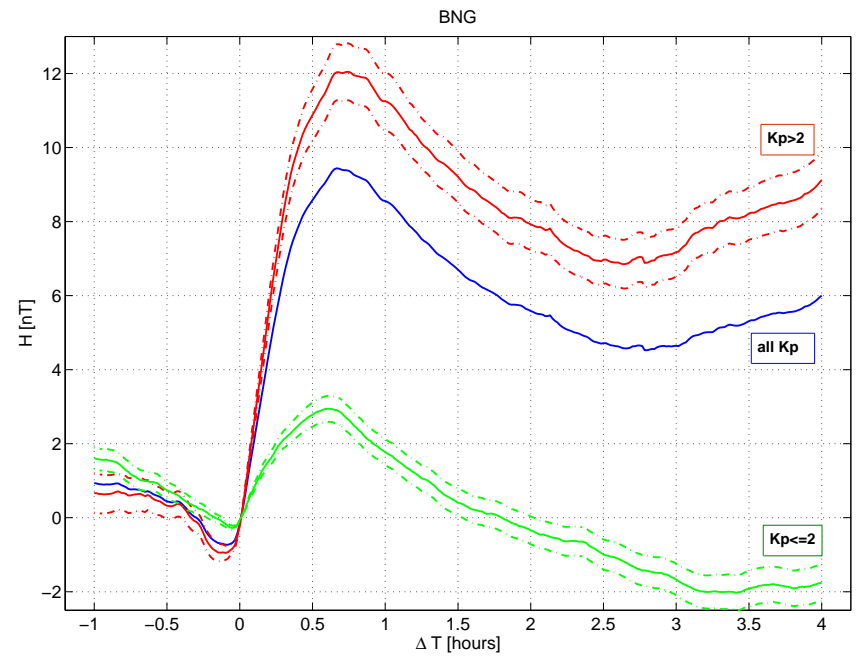

Fig. 7. Superposed Epoch Analysis of the recordings at the observatory BNG for all substorms (blue line), $K_{p}<=2$ (green lines) and $K_{p}>2$ (red lines). The dashed lines mark the $68.2 \%$ confidence intervals of the mean values (one standard deviation). The key time is $T_{0}$, the onset times of the substorm events.

the magnetic activity. It gets up to $12 \mathrm{nT}$ for events with $K_{p}>2$, whereas for small $K_{p}$ it hits only $3 \mathrm{nT}$. The blue and red curves show a second increase at times $>2.5 \mathrm{~h}$ indicating the onset of subsequent events.

As the substorm signature described by Caan et et al. (1975) were obtained using the data of several observatories at mid-latitudes, we compared our results with data from further observatories in the same time sector. Figure 8 shows the combined results of the superposed epoch analysis applied to the recordings of the four observations introduced in Sect. 2.2. The stations cover the latitudinal range from the equator to $52^{\circ}$ Mlat. Interestingly, despite the wide separation of the observatories, the magnetic deflections of the horizontal components, $H$, are practically identical during the substorm expansion phase. The peek amplitudes are almost the same. Differences are evident during the recovery phase: at higher latitudes, the signal decays faster. It is also interesting that the field strength starts rising already before the reported onset time, $T_{0}$, at all four observatories. On average the inflection point precedes $T_{0}$ by $3-4 \mathrm{~min}$. The comparison of these observations - with their similarities and differences - may help to constrain the concept of the substorm current system.

\subsection{Near-Earth observations - CHAMP}

The time span covered by the substorm catalog of Frey and Mende (2006) is well-overlapping with the high-quality magnetic field measurements of the CHAMP satellite. Thus it can be used broadly to perform similar statistics aiming to disclose substorm-related features also in satellite data. For this purpose the CHAMP data were corrected for the main

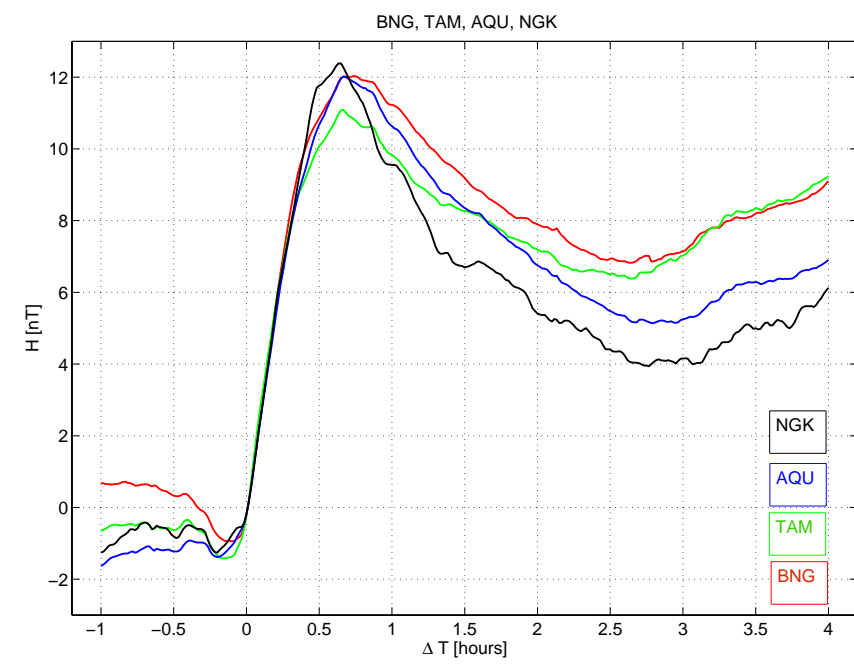

Fig. 8. Superposed Epoch Analysis at the observatories BNG $\left(4.36^{\circ}\right.$ Mlat, $90.8^{\circ}$ Mlon), TAM (24.8 ${ }^{\circ}$ Mlat, $81.6^{\circ}$ Mlon), AQL (42.45

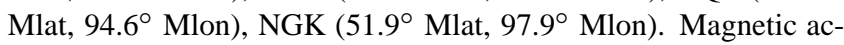
tivity level: $K_{p}>2$.

and crustal field as described for the single event at the beginning of Sect. 3.4. The average substorm signature in the magnetic field reveals itself quite easily in ground observations when time series of measurements are stacked using superposed epoch analysis. When looking into satellite data, however, the statistical approach is less straight-forward and data have to be selected carefully.

The data example presented in Fig. 4 already shows the difficulties that need to be handled when stacking and averaging the satellite recordings. To start with, the measurement is done from a moving observatory. As a result, the magnetometer readings contain a blend of time and spatial variations. Therefore, only data from a limited latitudinal interval can be stacked. We selected nighttime orbital arcs at latitudes between $-50^{\circ}$ to $50^{\circ}$ Mlat, clearly off the polar current systems. As we intended to compare data on the night side, we selected only those orbit intervals that were sampled in the local time sector 22:00-02:00 LT. The average local time for a substorm onset, $T_{0}$, is 23:00 $\mathrm{h}$ (Frey and Mende, 2006). Since $T_{0}$ marks the western footprint of the substorm FAC system, the LT interval chosen for stacking the data aims at catching orbits crossing the current wedge loop between the upward and the downward FAC paths connecting the tail current with the polar ionosphere.

In order to identify the peak amplitude variation due to the substorm reconfiguration, only orbital arcs before and after a substorm onset were compared. To obtain well-timed "after" arcs, substorm onsets had to occur in the time interval $1 \mathrm{~h}$ to $20 \mathrm{~min}$ before the satellite entered the subsequent latitudinal range $\pm 50^{\circ}$ Mlat on the night side. The satellite then needs approximately $25 \mathrm{~min}$ to cross the profile. The previous night-side pass, occurring $1.5 \mathrm{~h}$ earlier, was declared 


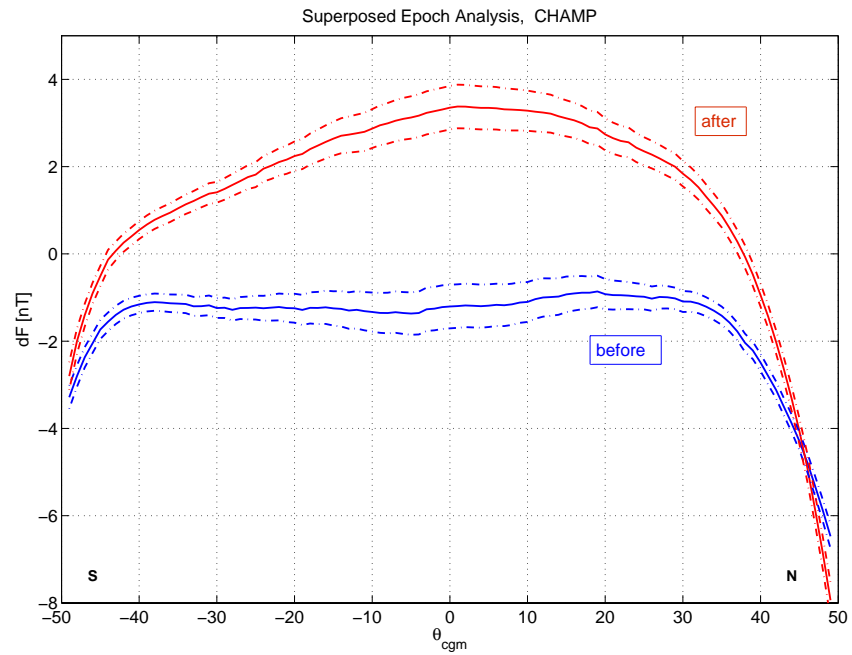

Fig. 9. CHAMP Superposed Epoch Analysis of the scalar residuals, $d F$, for all substorms. The blue line marks orbit sections before substorm onsets, the red line marks orbits after substorm onsets. Dashed lines mark the $68.2 \%$ confidence intervals of the mean values.

"before" pass. According to this definition CHAMP sampled the magnetic field variations at the nightside equator at some delay ranging between 32 and $72 \mathrm{~min}$ after $T_{0}$. As can be seen from the ground based measurements in Figs. 7 and 8, in that time interval the substorm-related field enhancement ranges between $90 \%-100 \%$ of its peak value. Thus, the satellite observations derived from the selected orbit arcs reflect maximum deflections due to a substorm at middle latitudes very well.

Following this procedure, 813 substorm events from the substorm catalog of Frey and Mende (2006) could be used for the CHAMP superposed epoch analysis. "Before" and "after" arcs were stacked separately. One of the selected events, for example, is the substorm shown in Fig. 5. Here, the blue and red curves were sorted into the "before" and "after" datasets, respectively. The resulting average latitude profiles of the signature in the scalar magnetic field residuals are shown in Fig. 9. Again, dashed lines mark the $68.2 \%$ confidence intervals of the respective mean curves. The red "after" curve is clearly shifted upward compared to the blue "before" curve. The shift is largest (about $4 \mathrm{nT}$ ) at the magnetic equator and decreases as the satellite moves towards the poles. It is worth noting that the shifts are distributed asymmetrically in both hemispheres: it amounts to $2 \mathrm{nT}$ in the northern half of the arc (right), and only to $1 \mathrm{nT}$ at southern latitudes (left).

The difference between the "before" and "after" curve reflects the latitudinal signature of a substorm reconfiguration. It is shown as the blue curve in Fig. 10. In this case, all available events are used irrespective of magnetic activity. The mean difference between the "before" and "after" curves,

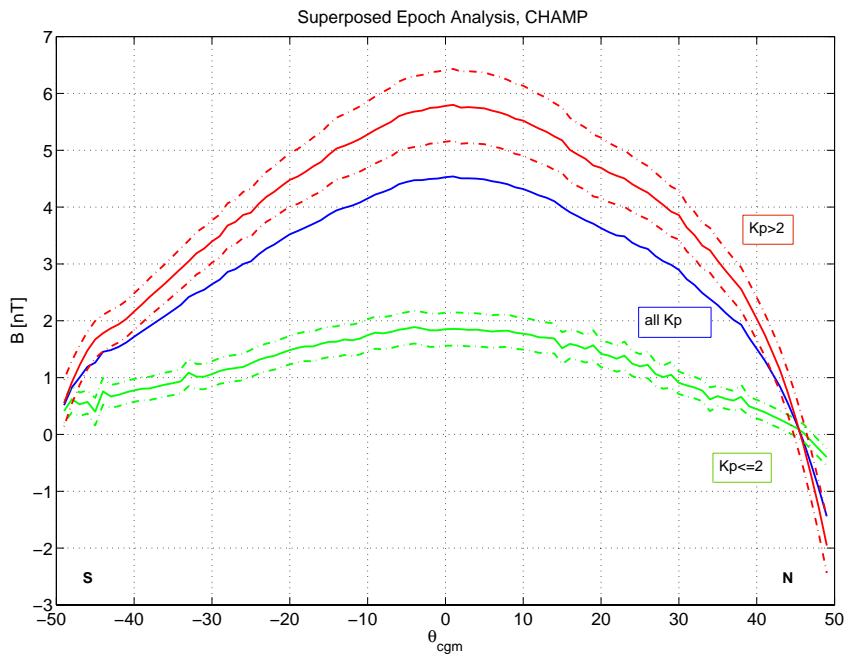

Fig. 10. Differences between the CHAMP scalar residuals, $d F$, before and after the substorm onsets. The activity level is color coded: all events (blue line, 813 events), $K_{p}<=2$ (green lines, 263 events) and $K_{p}>2$ (red lines, 550 events).

i.e. the average amplitude of the magnetic signature is largest $(4.5 \mathrm{nT})$ near the magnetic equator. As we concluded already for ground measurements in Sect. 4.1, the amplitude of the magnetic signature depends strongly on magnetic activity. In satellite measurements this can be observed, too; the green and red curves of Fig. 10 present the average shifts for low and high $K_{p}$ values, respectively. Events with low activity $\left(K_{p}<=2\right)$ show only a small enhancement of approximately $1.9 \mathrm{nT}$ whereas for higher $K_{p}>2$ the shift increases to almost $6 \mathrm{nT}$. Single events, like the example shown in Fig. 6, may exhibit much larger signatures (this event: $K_{p}=4-$, shift: $10 \mathrm{nT}$ ). Obviously, the same is true for the ground data.

Figure 11 presents the average substorm signature in the three magnetic field components $\left(B_{x}\right.$ northward, $B_{y}$ eastward, $B_{z}$ vertically downward, dipole coordinates) for enhanced magnetic activity, $K_{p}>2$. The red line repeats the variation of the scalar field signature of Fig. 10. The black curve reflecting the $B_{x}$-component displays an average positive displacement of about $6 \mathrm{nT}$. The amplitude changes little over the latitude range of $\pm 40^{\circ}$ and at the equator it accounts fully for the signature observed in the scalar field. The $B_{y}$ deflections change sign from negative to positive when the satellite passes from the southern to the Northern Hemisphere. The average $B_{z}$ deflection changes from positive to negative values when the satellite crosses the equator from south to north.

\section{Discussion}

The aim of this study is to identify the magnetic signature of a substorm in the near-Earth range at low and mid latitudes. We used the multitude of substorm onsets cataloged by Frey 


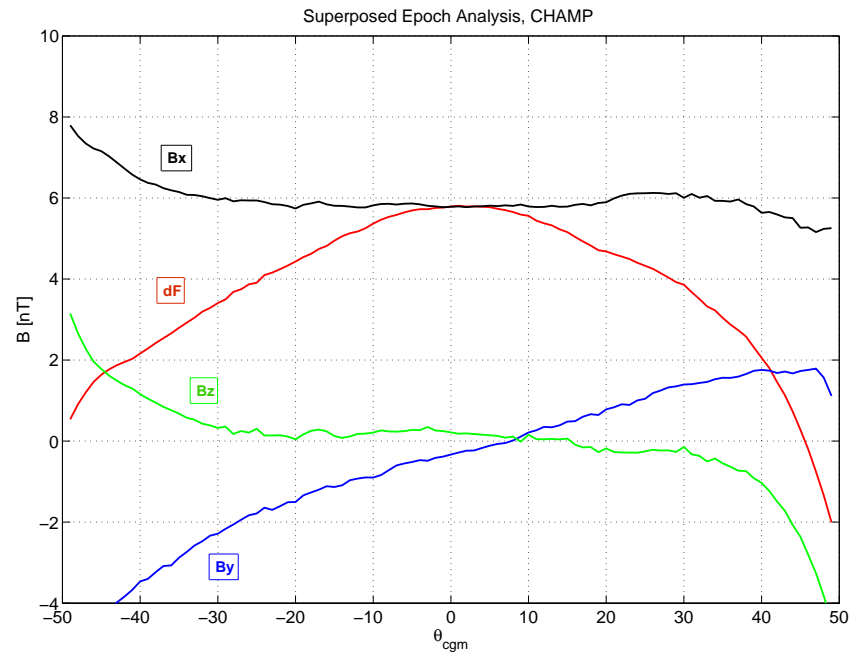

Fig. 11. Differences between the components before and after the substorm onsets: Red line: $d F$, black, blue and green lines mark $\left(B_{x}, B_{y}, B_{z}\right)$, respectively. The magnetic components are in dipole coordinates. Magnetic activity level: $K_{p}>2$.

and Mende (2006) covering a period from the middle of 2000 to the end of 2005. The typical magnetic signature of one of these substorms is shown for ground stations located at high and low latitudes and is compared to measurements of the CHAMP satellite along a mid- and low-latitudinal orbital arc. From this single example already characteristic features of the substorm-related magnetic variations could be deduced. As has been reported in many previous publications, at auroral latitudes a strong reduction of the northward component can be observed on ground which is caused by the westward flowing substorm electrojet. We will not discuss this wellknown high-latitude feature any further. Using all substorm events, we performed a statistical analysis of the ground and satellite observations at low and mid latitudes.

\subsection{Observed substorm-related signatures}

The average progression of the $H$-component of the nearequator station BNG resulting from a superposed epoch analysis of numerous night side measurements supports the substorm observations by Caan et al. (1975). The observed amplitude before, during and after the onset time $T_{0}$ reflects the processes associated with the current wedge formation during the substorm onset phase: the decreasing amplitude before the brightening of the aurora (i.e. in the loading phase) is due to a partial increase of the ring current (Clauer and McPherron, 1980; Hashimoto et al., 2002.) and/or an increase of the cross-tail current density just before the break up; the subsequent magnetic field enhancement stretching over $1 \mathrm{~h} 15 \mathrm{~min}$ is caused by the current disruption and redirection towards the polar ionosphere during the expansion phase. A second increase of the mean amplitude at times
$>2.7 \mathrm{~h}$ indicates the onset of consecutive substorms. This leads to a discussion of how the processes associated with the various substorm phases may be explained by the observations presented in this study.

In the observatory records in Fig. 8 we can see that the increase of the field strength starts 3-4 min before the reported onset time. This may partly be caused by a tardy estimate of the substorm onset from the two-min-spaced auroral images. On the other hand, the earlier increase may partly reflect the particle travel time from the tail to the high latitude ionosphere. In any case, the substorm initialization in the tail starts generally a few minutes before the onset time reported in the substorm catalog by Frey and Mende (2006); for an example see also Wang et al. (2008).

During the expansion phase the maximum amplitude of single events (e.g. Fig. 4) can be remarkably larger than the average magnetic deflection of approx. $9 \mathrm{nT}$. These large signatures usually coincide with high $K_{p}$ values (4- in our example) indicating elevated magnetic activity conditions during these events. The significant difference between the average magnetic deflections from data subsets with small and large $K_{p}$ values, as shown in Fig. 7, supports this assertion. These subsets still contain a large enough number of time series to yield significant results. The mean curve for low $K_{p}$ shows no secondary increase towards the end of the time interval. Obviously, at times of low magnetic activity, the time gap between substorms is larger than $4 \mathrm{~h}$. For higher magnetic activity the next substorm seems to follow after approximately $3 \mathrm{~h}$. This is consistent with the substorm recurrence period of $3 \mathrm{~h}$, as reported e.g. by Wang and Lühr (2007).

The superposed epoch analysis of the data from four observatories at similar longitudes (cf. Fig. 8) reveals that the onset-related deflection of the $H$ component is independent of latitude within a range of $0^{\circ}$ to $50^{\circ}$ Mlat. This is an interesting result, and it poses constraints on the geometry of the involved current systems. Below, we will come back to this point. During the recovery phase, however, the $H$ deflections show a latitudinal dependence. Here, the mid latitude stations experience a faster decay of the signal than observed in low latitude measurements.

For the CHAMP satellite data the superposed epoch analysis had to be performed along a latitudinal profile rather than over a time interval, as in the case of ground-based observations. Therefore, the two types of observations complement each other quite well. The difference of the $B_{x}$ average curves before and after substorm onsets yields the average magnetic signature as seen at satellite height. Although these profiles contain a certain mixture of temporal and spatial variations of the magnetic field, the obtained curve can be regarded the average latitude profile for the time of maximum substorm signal. In order to minimize the effect of unrelated time variation between the two profiles, corrections of magnetospheric and ring currents (reflected by $D_{s t}$ ) according to Maus and Lühr (2005) were applied to the satellite measurements. The spatial variations were minimized as far as 


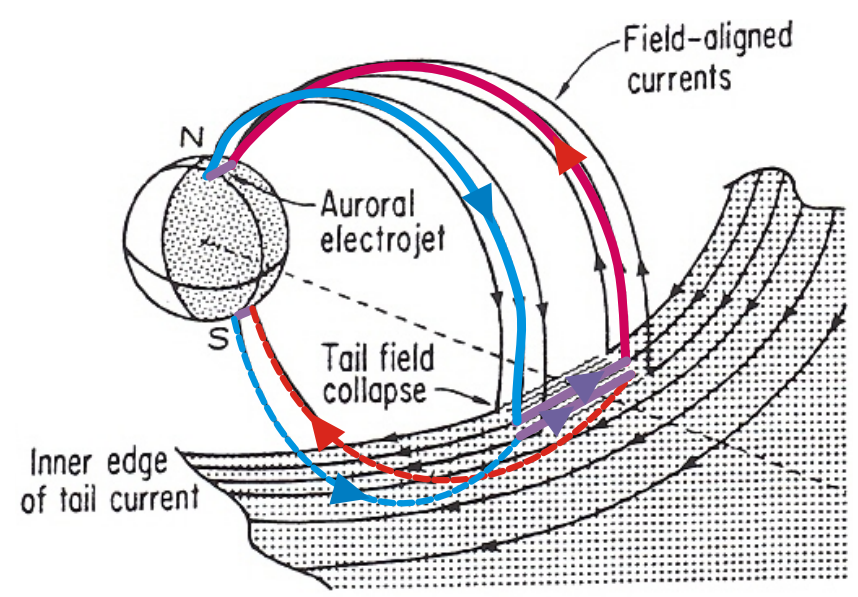

Fig. 12. Model of a current system that may explain the magnetic field due to the substorm current wedge (adopted from Clauer and McPherron, 1974). The colored lines sketch the assumed current loops.

possible by subtracting a recent crustal magnetic field model, MF5 (Maus et al., 2007), from the magnetic vector data.

One significant result of the CHAMP observations is that the amplitude of the northward component, $B_{x}$, is practically independent of latitude in the investigated range of $\pm 50^{\circ}$ Mlat. The deviation of the total field, $d F$, is, as expected, equal to the $B_{x}$ amplitude at the magnetic equator. Based on this relation, we may deduce the level of horizontal deflection from the peak value of $d F$ in Figs. 10 and 11. Also worth noting is the low level of spatial variation in the vertical component, $B_{z}$, within the latitude range $\pm 30^{\circ}$.

If we compare the signatures measured by CHAMP and on ground (e.g. Figs. 7 to 11), several interesting features emerge. Both systems indicate a latitude-independent deflection of the horizontal component during the expansion phase. But we also find that the satellite observations are smaller than the observatory results by a factor of 2 . Furthermore, there is a amplitude difference between the four observatories during the recovery phase. A suitable model of the current system should be able to reconcile all these different observations.

\subsection{The substorm current model}

In order to interpret the average magnetic signature that results from the superposed analyses of the satellite and observatory measurements we computed the response to the substorm current wedge model, as proposed by Clauer and McPherron (1974). For this purpose two closed current loops following the dipole field lines were placed in the magneto-

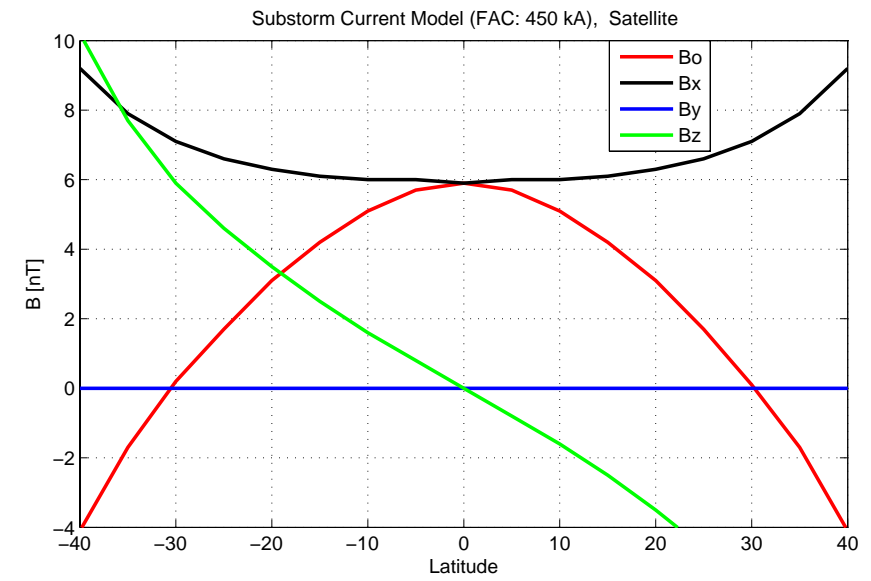

Fig. 13. Model results of the magnetic signatures along a simulated CHAMP orbit at the centre of the current system. Red line: $d F$, black, blue and green lines mark $\left(B_{x}, B_{y}, B_{z}\right)$, respectively.

sphere, diverting the cross-tail current at $7 R_{E}$ into the polar ionospheres of the Southern and Northern Hemispheres at $68^{\circ}$ of invariant latitude. The two FAC branches are separated by $2 \mathrm{~h}$ of local time. Figure 12 shows a sketch of this current configuration. A total current strength of $I=450 \mathrm{kA}$ was chosen in each loop to produce a magnetic field of 6 nT comparable to the mean observed signature at CHAMP (cf. Fig. 11). Figure 13 shows the predicted scalar field residuals (red curve) and the magnetic field components along a latitudinal profile $-40^{\circ} \ldots 40^{\circ}$ on a virtual orbit along the central magnetic meridian within the loop. The colour codes of $B_{x}, B_{y}$, and $B_{z}$ are the same as for the satellite measurements presented in Fig. 11.

The amplitude of the scalar field effect attains a maximum at the equator and decreases towards the poles. Its shape is similar to the measured field. Different from the simulated values, the measured scalar field exhibits a slower decrease towards the poles and the zero-crossings occur at higher latitudes $\left(-50^{\circ}\right.$ and $\left.45^{\circ}\right)$. The modelled $B_{x}$ component, carrying the major part of the onset-related magnetic field signal, is also quite consistent with the observed $B_{x}$ : it is constant over a latitudinal range of $\pm 20^{\circ}$ off the magnetic equator at an amplitude of approximately $6 \mathrm{nT}$. Towards higher latitudes, however, the modelled horizontal field increases much faster than the measurements. As expected, the modelled $B_{y}$ equals zero along the complete profile because the virtual orbit is centered exactly between the upward and downward arcs of the current reconfiguration loop. The measured $B_{y}$ component, however, runs from negative to positive values from the Southern to the Northern Hemisphere. This difference may be due to the fact that the actual orbits contributing to the average curve are not well-centered between the two FAC branches of the substorm current loop, and implies that the mean sampling may take place closer to the pre-midnight upward FACs. The $B_{z}$ deflection changes sign in opposite 
direction, from positive to negative values on the satellite's northbound path across the equator. The measured $B_{z}$ shows the same trend, but has a more gentle slope than the modelled component.

From this comparison we may conclude that the simple substorm current model can predict the signatures generated during an onset only qualitatively but fails to account for the details.

\subsection{An improved substorm current model}

In order to reconcile the apparently conflicting results we extend the simple current model of Fig. 12. The amplitude difference between the satellite and ground-based observations implies the existence of ionospheric currents. An eastward flowing ionospheric current generates a northward directed magnetic field on ground and a southward directed field at satellite height. Such currents might explain the average difference of $6 \mathrm{nT}$ in the measurements shown in Figs. 8 and 11 (see also the single event displayed in Figs. 4 and 6). It is known that the ionospheric conductivity is low during the night, but some residual currents may still flow. The question arises, what could be the driver for the eastward current at middle latitudes? At high latitudes, the primary substormrelated electric field points from dawn to dusk. For the midand low-latitude currents an oppositely directed E-field is required. Therefore, we assume the existence of an accompanying Region- 2 current system that can support eastward currents when over-shielding occurs and the electric field penetrates to lower latitudes.

Another open issue is the deflection difference of the $H$ components at the four observatories during expansion and recovery phases. This can also not be explained by the simple current model of Fig. 12. Our interpretation approach is to introduce two different sources of the magnetic field: (a) the reduction of the tail lobe field strength and (b) the magnetic field originating from the current wedge circuit. For an estimate of the total current flowing in this circuit we may take into consideration observations at auroral latitudes. In the case of the example presented in this study, station MAS (cf. Fig. 3) reflects the strength of the substorm electrojet quite well. During the intensification of the electrojet, the field strength at the low-latitude station BNG increases (cf. Fig. 4) in phase with the high latitude deflection. Both observations reach their peaks at the same time. This implies that both stations respond to the same current circuit. However, when the electrojet dies out at 23:00 UT still a significant deflection of $15 \mathrm{nT}$ can be observed at the equator. This brings the second magnetic field source into play: the influence of the tail lobe field. The magnetic field is primarily generated by cross-tail currents which close via the northern and southern lobes. Since part of the cross-tail current is diverted into the ionosphere the tail field is reduced. After the onset stations at low and mid latitudes record the effect of the increase in the substorm current circuit and the decay of the tail lobe field strength at the same time. Both provide northward contributions. When the substorm currents cease only the effect of the lobe field is observable.

In a statistical study, Maus and Lühr (2005) found that the tail lobe magnetic field causes a southward directed magnetic field at the Earth's surface that is well-aligned with the GSM $\mathrm{Z}$-component. When averaged over several years it appears to be aligned with the magnetic dipole axis. Thus, the response of the $H$ component to the tail lobe magnetic field should be latitude-dependent. Its variation follows the cosine of magnetic latitude. When looking at the $H$ deflections displayed in Fig. 8, approximately $2.7 \mathrm{~h}$ after the onset the differences at the four observatories can well be explained by the cosine law. This supports our idea that only the decreased tail lobe field is observed here.

Based on this new concept we propose a more detailed model for describing the substorm-related magnetic signatures. It combines three elements: the substorm current circuit, the tail lobe magnetic field decay and the shielding Region-2 FACs with their closing ionospheric current at low and mid latitudes. In a series of model runs, we try to reproduce the situation at the time of peak deflection, about three-quaters of an hour after the onset. As an example we take the mean situation for enhanced activity as presented in Figs. 8 and 11. We pick the amount of reduced tail lobe field effect after the substorm from the $H$ deflection at BNG at a time $2.7 \mathrm{~h}$ after the onset (cf. Fig. 8). Its value is about $7 \mathrm{nT}$. At the time of peak deflection $(\Delta t=0.72 \mathrm{~h})$ all three contributions add up. At all observatories we obtain values of approximately $12 \mathrm{nT}$, while CHAMP records only $6 \mathrm{nT}$ at that time (cf. Fig. 11). In order to explain this difference an eastward ionospheric current covering the latitude range of $\pm 50^{\circ}$ Mlat is introduced. The current density required at low latitudes is $5 \mathrm{~mA} / \mathrm{m}$, a rather weak current. Finally we adjust the main current in the substorm circuit to match the deflections at the observatories and CHAMP. To achieve this together with the described intensities of the other two magnetic field sources we need a total current of $300 \mathrm{kA}$ to reproduce the substorm scenery at elevated magnetic activity.

If we apply these values to our substorm current model a satisfactory agreement with the observations close to the equator is reached. However, there are significant deviations from the observations at mid latitudes (not shown). In order to obtain a good agreement at all latitudes, on ground as well as at satellite height, we have to reduce the magnetospheric field variation at the time of peak deflection to $5 \mathrm{nT}$ and enhance the total current of the substorm FACs to $360 \mathrm{kA}$. A Region-2 FAC system with a total current of $120 \mathrm{kA}$ is installed at invariant latitudes around $60^{\circ}$ to feed the eastward ionospheric currents. The associated partial ring current, as a fourth model element, reduces the effect of the tail lobe current change by $2 \mathrm{nT}$ on the ground. Figure 14 shows the latitudinal profiles of the modelled magnetic field components both at CHAMP altitude and on ground at the time of peak deflection, about $40 \mathrm{~min}$ after the onset. With these improved 

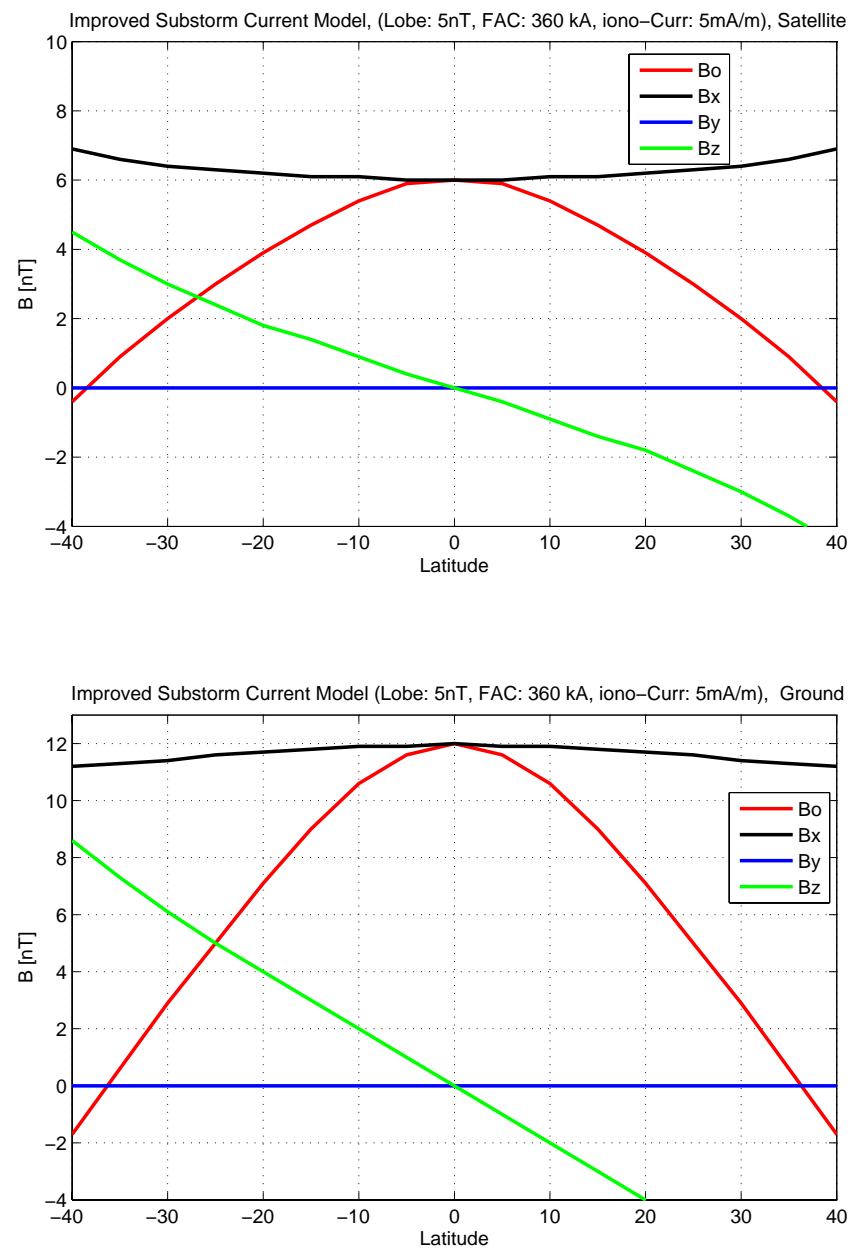

Fig. 14. Improved model: results of the magnetic signatures at CHAMP altitude (top frame) and on the ground (bottom frame) at the time of peak deflection, about $40 \mathrm{~min}$ after the onset. Red line: $d F$, black, blue and green lines mark $\left(B_{x}, B_{y}, B_{z}\right)$, respectively.

model parameters all features observed in the measurements are reproduced well.

What do these new model parameters imply? We regard the adjustment of the total magnetospheric field for our final model estimates justified. The value of $7 \mathrm{nT}$ for the tail lobe field change, as determined $2.7 \mathrm{~h}$ after the onset, is valid when the ring current has decayed. However, it is too high to describe the situation at the time of peak deflection $\Delta t=0.7 \mathrm{~h}$. A completely new element in the substorm scenario is the presence of eastward ionospheric currents at mid and low latitudes. For their explanation we introduced a R-2 FAC system at $60^{\circ}$ of invariant latitude connected to the ring current that provides an over-shielding of the auroral electric field. This configuration is justified by the observations shown in the selected substorm example. In Fig. 3 the subauroral station TAR shows a positive deflection of $30 \mathrm{nT}$ in the north component during the substorm. This indicates the presence of eastward currents at subauroral latitudes. The

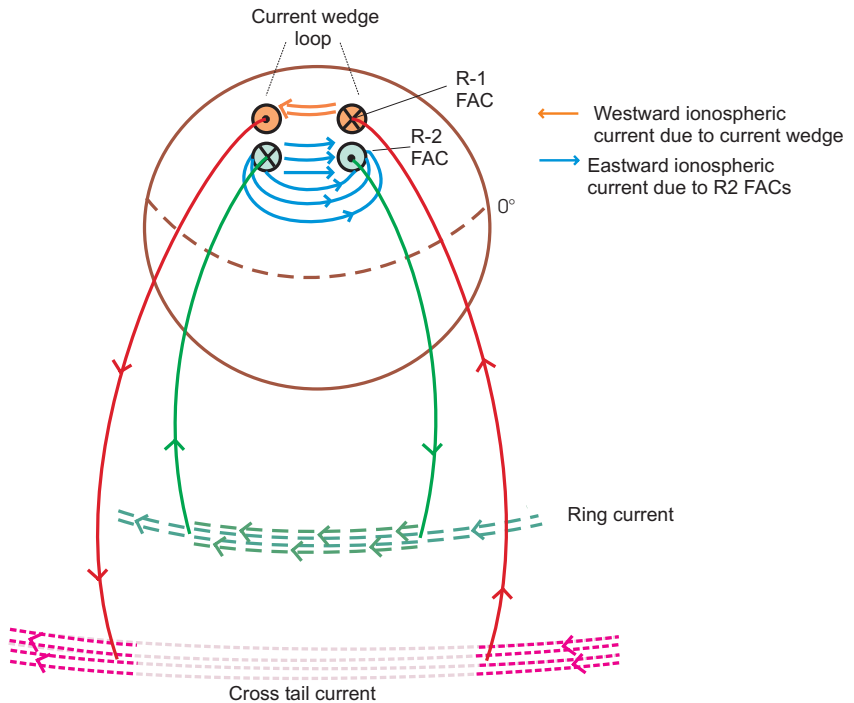

Fig. 15. Improved model of a current system displaying the closure of the current wedge loop in the ionosphere (orange-red color scheme) and the eastward electrojet due to the equatorward Region2 FACs and enhanced ring-current (blue-green color scheme).

presence of eastward ionospheric currents at mid latitudes in the context of substorms was reported earlier by Feldstein et al. (1997). From our model we envisage an ionospheric current configuration as sketched in Fig. 15. For the total current diverted from the current wedge into each hemisphere we obtained $360 \mathrm{kA}$. This seems to be less than the total current in the substorm electrojet. Obviously, an additional part of the electrojet is closed as a toroidal current in the ionosphere. For the R-2 FAC we had to use $120 \mathrm{kA}$ to achieve a good fit with the observations. These R-2 FACs are believed to be driven by the pressure gradient in the near-Earth tail that is build-up by the Earthward plasma flow during the course of a substorm (e.g. Antonova, 2004).

The details of the current systems at high latitudes have not been studied here. They should be the target of a dedicated research effort in order to obtain a consistent picture of substorm-related effects both at high and low latitudes.

\section{Conclusions}

In this study we provide a consistent picture of the near-Earth current systems associated with magnetospheric substorms. The main features are derived from the average magnetic deflections observed at mid and low latitudes after substorm onsets. The constrains inferred from simultaneous groundbased and satellite measurements enable us to design a detailed current system. For one example we show the consistency of this configuration even with high latitude magnetic field recordings.

Important features of the substorm-related magnetic variations are: 
1. During the substorm expansion phase there is a latitudeindependent deflection of the horizontal component $H$ in the $\pm 50^{\circ}$ Mlat range.

2. During the recovery phase the decay of the deflection is slowest near the equator.

3. At CHAMP altitude $(\approx 400 \mathrm{~km})$ the $H$ component deflection is only half as strong as on ground.

The current system proposed to explain the magnetic field variations consists of four elements:

1. The current wedge loop routing part of the cross-tail current through the ionosphere,

2. The changing tail lobe current becoming weaker over the course of a substorm,

3. An eastward ionospheric current covering the whole latitudinal range from the subauroral region to the equator.

4. A partial ring current connected to the Region-2 FACs.

The nightside ionospheric current (3) has never been considered previously. Although being rather weak (only a few $\mathrm{mA} / \mathrm{m}$ ) this current is needed to explain the amplitude difference between ground-based and satellite measurements.

According to our model these four elements contribute with different percentages to the observed magnetic signals. During the expansion phase they all add up, and at the time of peak deflection (12nT) we may attribute $7 \mathrm{nT}(57 \%)$ to the lobe field strength variation, $4 \mathrm{nT}(33 \%)$ to the current wedge loop, $3 \mathrm{nT}(25 \%)$ to the ionospheric current, and $-2 \mathrm{nT}(-15 \%)$ to the partial ring current. Interestingly, only one-third of the near-Earth substorm signature is caused by the re-routing of the tail current. At approximately $2.7 \mathrm{~h}$ after the onset only the change in lobe field strength is recorded while the other currents have ceased.

A topic not studied here is the connection to related auroral currents and their consistency with the proposed current model. This may be addressed in a follow-up study.

The results of our investigations are of particular interest also for main field modelling since there is no correction of the employed data for substorm effects so far. Our statistics show that a selection by low $K_{p}$ values reduces the amplitude of unwanted substorm signatures in the CHAMP magnetic data to a large extend. The best approach, however, would be to omit data of the time interval starting from substorm onsets to $2 \mathrm{~h}$ after $T_{0}$.

Acknowledgement. We are obliged to H. Frey of SWRI for making available the catalog of auroral substorm onsets obtained from IMAGE-FUV observations. We also thank S. Mende for providing the online FUV images of our example shown in Fig. 2. The operational support of the CHAMP mission by the German Aerospace Center (DLR) and the financial support for the data processing by the Federal Ministry of Education (BMBF), as part of the Geotechnology Programme, are gratefully acknowledged.
Topical Editor M. Pinnock thanks T. Kikuchi and X. Cai for their help in evaluating this paper.

\section{References}

Antonova, E. E.: Magnetostatic equilibrium and current systems in the Earth's magnetosphere, Adv. Space Res., 33, 757-760, 2004.

Baker, D. N., Pulkkinen, T. I., Angelopoulos, V., Baumjohann, W., and McPherron, R. L.: Neutral line model of substorms: Past results and present view, J. Geophys. Res., 101, 12975-13010, 1996.

Caan, M. N., McPherron, R. L., and Russell, C. T.: Substorm and interplanetary magnetic field effects on the geomagnetic tail lobes, J. Geophys. Res., 80, 191-194, 1975.

Clauer, C. R. and McPherron, R. L.: Mapping of local time, universal time development of magnetosphere substorms using midlatitude magnetic observations, J. Geophys. Res., 79, 2812-2820, 1974.

Clauer, C. R. and McPherron, R. L.: The Relative Importance of the Interplanetary Electric Field and Magnetospheric Substorms on Partial Ring Current Development, J. Geophys. Res., 85(A12), 6747-6759, 1980.

Feldstein, Y. I., Grafe, A., Gromova, L. I., and Popov, V. A.: Auroral electrojets during geomagnetic storms, J. Geophys. Res., 102(A7), 14 223-14 235, 1997.

Frey, H. U. and Mende, S. B.: Substorm onsets as observed by IMAGE-FUV, Int. Conf. Substorms, 8, 71-75, 2006.

Hashimoto, K. K., Kikuchi, T., and Ebihara, Y.: Response of the magnetospheric convection to sudden interplanetary magnetic field changes as deduced from the evolution of partial ring currents, J. Geophys. Res., 107(A11), 1337, 2002.

Lopez, R. E., Lühr, H., Anderson, B. J., Newell, P. T., and Mcentire, R. W.: Multipoint Observations of a Small Substorm, J. Geophys. Res., 95, 18 897-18912, 1990.

Lühr, H., J. Warnecke, J., and Rother, M.: An algorithm for estimating field-aligned currents from single spacecraft magnetic field measurements: a diagnostic tool applied to Freja satellite data, IEEE Trans. Geosci. Remote Sens., 34, 1369-1376, 1996.

Lui, A. T. Y.: Current disruption in the Earth's magnetosphere: Observations and models, J. Geophys. Res., 101, 13 067-13088, 1996.

Maus, S. and Lühr, H.: Signature of the quite-time magnetospheric magnetic field and its electromagnetic induction in the rotating earth, Geophys. J. Int., 162(3), 755-763, 2005.

Maus, S., Rother, M., Stolle, C., Mai, W., Choi, S., Lühr, H., Cooke, D., and Roth, C.: Third generation of the Potsdam Magnetic Model of the Earth (POMME), Geochem. Geophys. Geosyst., 7, Q07008, doi:10.29/2006GC001269, 2006.

Maus, S., Lühr, H., Rother, M., Hemant, K., Balasis, G., Ritter, P., and Stolle, C.: Fifth generation lithospheric magnetic field model from CHAMP satellite measurements, Geochem. Geophys. Geosyst., 8, Q05013, doi:10.1029/2006GC001521, 2007.

Reigber, C., Lühr, H., and Schwintzer, P.: CHAMP mission status, Adv. Space Res., 30(2), 129-134, 2002.

Richmond, A. D.: Ionospheric electrodynamics using magnetic apex coordinates, J. Geomag. Geoelectr., 47, 191-212, 1995.

Sillanpää, I. H., Lühr, Viljanen, A., and Ritter, P.: Quiet-time Magnetic Variations at High Latitude Observatories, Earth Planets Space, in press, 56(1), 39-45, 2004. 
Wang, H. and Lühr, H.: Seasonal-longitudinal variation of substorm occurrence frequency: Evidence for ionospheric control, Geophys. Res. Lett., 34, L07104, doi:10.1029/2007GL029423, 2007.

Wang, H., Lühr, H., Ma, S.-Y., and Ritter, P.: Statistical study of the substorm onset: its dependence on solar wind parameters and solar illumination, Ann. Geophys., 23, 2069-2079, 2005,

http://www.ann-geophys.net/23/2069/2005/.
Wang, H., Lühr, H., Ma, S.-Y., and Frey, H.: Interhemispheric comparison of average substorm onset locations: evidence for deviation from conjugacy, Ann. Geophys., 25, 989-999, 2007, http://www.ann-geophys.net/25/989/2007/.

Wang, H., Lühr, H., Ma, S.-Y., and Ridley, A.: Substorm onset dynamics in the magnetotail as derived from joint TC-1 and Cluster data analysis, Earth Planets Space, 60, 613-621, 2008. 